\title{
Rigidity of area-minimizing two-spheres in three-manifolds
}

\author{
Hubert Bray, Simon Brendle and Andre Neves
}

We give a sharp upper bound for the area of a minimal two-sphere in a three-manifold $(M, g)$ with positive scalar curvature. If equality holds, we show that the universal cover of $(M, g)$ is isometric to a cylinder.

\section{Introduction}

A classical result in differential geometry due to Toponogov [14] states that every simple closed geodesic $\gamma$ on a two-dimensional surface $(\Sigma, g)$ satisfies

$$
\operatorname{length}(\gamma)^{2} \inf _{\Sigma} K \leq 4 \pi^{2}
$$

where $K$ denotes the Gaussian curvature of $\Sigma$. Moreover, equality holds if and only if $(\Sigma, g)$ is isometric to the standard sphere $S^{2}$ up to scaling (see also [8] for a different proof).

We next consider a three-manifold $(M, g)$ with positive scalar curvature. By a theorem of Schoen and Yau [13], any area-minimizing surface in $M$ is homeomorphic to either $S^{2}$ or $\mathbb{R P}^{2}$. The case of area-minimizing projective planes was studied in [3]. In particular, if $\Sigma$ is an embedded $\mathbb{R P}^{2}$ of minimal area, then the area of $\Sigma$ can be estimated from above by

$$
\operatorname{area}(\Sigma, g) \inf _{M} R \leq 12 \pi
$$

(cf.[3, Theorem 1]). Moreover, equality holds if and only if $(M, g)$ is isometric to $\mathbb{R P}^{3}$ up to scaling. A survey of related rigidity results involving scalar curvature can be found in [4].

In this paper, we consider the case of area-minimizing two-spheres. We shall assume throughout that $(M, g)$ is a compact three-manifold with $\pi_{2}(M) \neq 0$. We denote by $\mathscr{F}$ the set of all smooth maps $f: S^{2} \rightarrow M$ which represent a non-trivial element of $\pi_{2}(M)$. We define

$$
\mathscr{A}(M, g)=\inf \left\{\operatorname{area}\left(S^{2}, f^{*} g\right): f \in \mathscr{F}\right\} .
$$

We now state the main result of this paper: 
Theorem 1.1. We have

$$
\mathscr{A}(M, g) \inf _{M} R \leq 8 \pi
$$

where $R$ denotes the scalar curvature of $(M, g)$. Moreover, if equality holds, then the universal cover of $(M, g)$ is isometric to the standard cylinder $S^{2} \times$ $\mathbb{R}$ up to scaling.

Inequality (1.2) follows directly from the formula for the second variation of area. We now describe the proof of the rigidity statement. By scaling, we may assume that $\mathscr{A}(M, g)=4 \pi$ and $\inf _{M} R=2$. It follows from results of Meeks and Yau [12] that the infimum in (1.1) is attained by a smooth immersion $f \in \mathscr{F}$ (see also [9]). Using the implicit function theorem, we construct a one-parameter family of immersed two-spheres with constant mean curvature. Using the formula for the second variation of area, we are able to show that these surfaces all have area $\mathscr{A}(M, g)=4 \pi$. Consequently, these spheres are all round and totally geodesic. This allows us to construct a local isometry from the cylinder $S^{2} \times \mathbb{R}$ into $M$. The use of constant mean curvature surfaces is motivated in part by work of Bray [1] and Huisken and Yau [11] (see also [2, 10]).

We note that Cai and Galloway [5] have obtained a similar rigidity theorem for minimal tori in three-manifolds of nonnegative scalar curvature. The proof in [5] uses a different argument based on a deformation of the metric to strictly positive scalar curvature. The arguments in this paper can be adapted to give an alternative proof of Theorem 1 in [5]. See also [7] for related work in this direction.

\section{Proof of (1.2)}

Let us consider a smooth immersion $f: S^{2} \rightarrow M$. Since $f$ has trivial normal bundle, there exists a globally defined unit normal field $\nu$. In other words, for each point $x \in S^{2}, \nu(x) \in T_{f(x)} M$ is a unit vector which is orthogonal to the image of $d f_{x}: T_{x} S^{2} \rightarrow T_{f(x)} M$. The following result is a consequence of the Gauss-Bonnet theorem.

Proposition 2.1. For any immersion $f: S^{2} \rightarrow M$, we have

$$
\int_{S^{2}}\left(R-2 \operatorname{Ric}(\nu, \nu)-|I I|^{2}\right) d \mu_{f^{*} g} \leq 8 \pi,
$$

where II denotes the second fundamental form of $f$. 
Proof. By the Gauss equations, we have

$$
R-2 \operatorname{Ric}(\nu, \nu)-|I I|^{2}=2 K-H^{2}
$$

where $H$ and $K$ denote the mean curvature and the Gaussian curvature, respectively. This implies

$$
\int_{S^{2}}\left(R-2 \operatorname{Ric}(\nu, \nu)-|I I|^{2}\right) d \mu_{f^{*} g} \leq 2 \int_{S^{2}} K d_{f^{*} g}=8 \pi
$$

by the Gauss-Bonnet theorem.

We next consider a map $f \in \mathscr{F}$ which attains the infimum in (1.1). The existence of a minimizer is guaranteed by the following result.

Proposition 2.2. There exists a smooth map $f \in \mathscr{F}$ such that area $\left(S^{2}, f^{*} g\right)=\mathscr{A}(M, g)$. Moreover, the map $f$ is an immersion.

Proposition 2.2 is an immediate consequence of Theorem 7 in [12] (see also [9, Theorem 4.2]). In fact, Meeks and Yau show that either $f$ is an embedding or a two-to-one covering map whose image is an embedded $\mathbb{R P}^{2}$. We will not use this stronger statement here.

Let $f \in \mathscr{F}$ be a smooth immersion with area $\left(S^{2}, f^{*} g\right)=\mathscr{A}(M, g)$. Using the formula for the second variation of area, we obtain

$$
\int_{S^{2}}\left(\operatorname{Ric}(\nu, \nu)+|I I|^{2}\right) u^{2} d \mu_{f^{*} g} \leq \int_{S^{2}}|\nabla u|_{f^{*} g}^{2} d \mu_{f^{*} g}
$$

for every smooth function $u: S^{2} \rightarrow \mathbb{R}$. Choosing $u=1$ gives

$$
\int_{S^{2}}\left(\operatorname{Ric}(\nu, \nu)+|I I|^{2}\right) d \mu_{f^{*} g} \leq 0 .
$$

Using Proposition 2.1, we obtain

$$
\begin{aligned}
\operatorname{area}\left(S^{2}, f^{*} g\right) \inf _{M} R & \leq \int_{S^{2}}\left(R+|I I|^{2}\right) d \mu_{f^{*} g} \\
& \leq 8 \pi+2 \int_{S^{2}}\left(\operatorname{Ric}(\nu, \nu)+|I I|^{2}\right) d \mu_{f^{*} g} \\
& \leq 8 \pi .
\end{aligned}
$$

This completes the proof of (1.2). 


\section{The case of equality}

In this section, we analyze the case of equality. Suppose that

$$
\mathscr{A}(M, g) \inf _{M} R=8 \pi
$$

After rescaling the metric if necessary, we may assume that $\mathscr{A}(M, g)=4 \pi$ and $\inf _{M} R=2$. By Proposition 2.2, we can find a smooth immersion $f \in \mathscr{F}$ such that area $\left(S^{2}, f^{*} g\right)=4 \pi$.

Proposition 3.1. Let $f \in \mathscr{F}$ be a smooth immersion such that area $\left(S^{2}, f^{*} g\right)=4 \pi$. Then the surface $\Sigma=f\left(S^{2}\right)$ is totally geodesic. Moreover, we have $R=2$ and $\operatorname{Ric}(\nu, \nu)=0$ at each point on $\Sigma$.

Proof. By assumption, we have area $\left(S^{2}, f^{*} g\right)=4 \pi$ and $\inf _{M} R=2$. Consequently, the inequalities in (2.1) are all equalities. In particular, we have

$$
\int_{S^{2}}\left(R+|I I|^{2}\right) d \mu_{f^{*} g}=8 \pi
$$

and

$$
\int_{S^{2}}\left(\operatorname{Ric}(\nu, \nu)+|I I|^{2}\right) d \mu_{f^{*} g}=0 .
$$

It follows from (3.2) that the constant functions lie in the nullspace of the Jacobi operator $L=\Delta_{f^{*} g}+\operatorname{Ric}(\nu, \nu)+|I I|^{2}$. This implies

$$
\operatorname{Ric}(\nu, \nu)+|I I|^{2}=0
$$

at each point on $\Sigma$. Moreover, since area $\left(S^{2}, f^{*} g\right)=4 \pi$ and $\inf _{M} R=2$, the identity (3.1) implies that $R=2$ and $|I I|^{2}=0$ at each point on $\Sigma$. This completes the proof.

Proposition 3.2. Let $f \in \mathscr{F}$ be a smooth immersion such that area $\left(S^{2}, f^{*} g\right)=4 \pi$. Then there exists a positive real number $\delta_{1}$ and a smooth map $w: S^{2} \times\left(-\delta_{1}, \delta_{1}\right) \rightarrow \mathbb{R}$ with the following properties:

- For each point $x \in S^{2}$, we have $w(x, 0)=0$ and $\left.\frac{\partial}{\partial t} w(x, t)\right|_{t=0}=1$.

- For each $t \in\left(-\delta_{1}, \delta_{1}\right)$, we have $\int_{S^{2}}(w(\cdot, t)-t) d \mu_{f^{*} g}=0$. 
- For each $t \in\left(-\delta_{1}, \delta_{1}\right)$, the surface

$$
\Sigma_{t}=\left\{\exp _{f(x)}(w(x, t) \nu(x)): x \in S^{2}\right\}
$$

has constant mean curvature.

Proof. The Jacobi operator associated with the minimal immersion $f: S^{2} \rightarrow$ $M$ is given by $L=\Delta_{f^{*} g}+\operatorname{Ric}(\nu, \nu)+|I I|^{2}$. Using Proposition 3.1, we conclude that $L=\Delta_{f^{*} g}$. Hence, the assertion follows from the implicit function theorem.

For each $t \in\left(-\delta_{1}, \delta_{1}\right)$, we define a map $f_{t}: S^{2} \rightarrow M$ by $f_{t}(x)=\exp _{f(x)}$ $(w(x, t) \nu(x))$. Clearly, $f_{0}(x)=f(x)$ for all $x \in S^{2}$. To fix notation, we denote by $\nu_{t}(x) \in T_{f_{t}(x)} M$ the unit normal vector to the surface $\Sigma_{t}=f_{t}\left(S^{2}\right)$ at the point $f_{t}(x)$. We assume that $\nu_{t}$ depends smoothly on $x$ and $t$, and $\nu_{0}(x)=$ $\nu(x)$ for all $x \in S^{2}$. Moreover, we denote by $I I_{t}$ the second fundamental form of $f_{t}$.

Lemma 3.3. There exists a positive real number $\delta_{2}<\delta_{1}$ with the following property: if $t \in\left(-\delta_{2}, \delta_{2}\right)$ and $u: S^{2} \rightarrow \mathbb{R}$ is a smooth function satisfying $\int_{S^{2}} u d \mu_{f_{t}^{*} g}=0$, then

$$
\int_{S^{2}}|\nabla u|_{f_{t}^{*} g}^{2} d \mu_{f_{t}^{*} g}-\int_{S^{2}}\left(\operatorname{Ric}\left(\nu_{t}, \nu_{t}\right)+\left|I I_{t}\right|^{2}\right) u^{2} d \mu_{f_{t}^{*} g} \geq 0 .
$$

Proof. We can find a uniform constant $c>0$ such that

$$
\int_{S^{2}}|\nabla u|_{f_{t}^{*} g}^{2} d \mu_{f_{t}^{*} g} \geq c \int_{S^{2}} u^{2} d \mu_{f_{t}^{*} g}
$$

for each $t \in\left(-\delta_{1}, \delta_{1}\right)$ and every smooth function $u: S^{2} \rightarrow \mathbb{R}$ satisfying $\int_{S^{2}} u d \mu_{f_{t}^{*} g}=0$. Moreover, it follows from Proposition 3.1 that

$$
\sup _{S^{2}}\left(\operatorname{Ric}\left(\nu_{t}, \nu_{t}\right)+\left|I I_{t}\right|^{2}\right) \rightarrow 0
$$

as $t \rightarrow 0$. Putting these facts together, the assertion follows.

Lemma 3.4. For each $t \in\left(-\delta_{1}, \delta_{1}\right)$, we have

$$
\int_{S^{2}}\left(\operatorname{Ric}\left(\nu_{t}, \nu_{t}\right)+\left|I I_{t}\right|^{2}\right) d \mu_{f_{t}^{*} g} \geq 0
$$


Proof. Since $f$ minimizes area in its homotopy class, we have

$$
\operatorname{area}\left(S^{2}, f_{t}^{*} g\right) \geq \operatorname{area}\left(S^{2}, f^{*} g\right)=4 \pi \text {. }
$$

Moreover, we have $\inf _{M} R=2$. Applying Proposition 2.1 to the map $f_{t}$ : $S^{2} \rightarrow M$, we obtain

$$
\begin{aligned}
8 \pi & \leq \operatorname{area}\left(S^{2}, f_{t}^{*} g\right) \inf _{M} R \\
& \leq \int_{S^{2}}\left(R+\left|I I_{t}\right|^{2}\right) d \mu_{f_{t}^{*} g} \\
& \leq 8 \pi+2 \int_{S^{2}}\left(\operatorname{Ric}\left(\nu_{t}, \nu_{t}\right)+\left|I I_{t}\right|^{2}\right) d \mu_{f_{t}^{*} g} .
\end{aligned}
$$

From this, the assertion follows.

By assumption, the surface $\Sigma_{t}$ has constant mean curvature. The mean curvature vector of $\Sigma_{t}$ can be written in the form $-H(t) \nu_{t}$, where $H(t)$ is a smooth function of $t$. For each $t \in\left(-\delta_{1}, \delta_{1}\right)$, the lapse function $\rho_{t}: S^{2} \rightarrow \mathbb{R}$ is defined by

$$
\rho_{t}(x)=\left\langle\nu_{t}(x), \frac{\partial}{\partial t} f_{t}(x)\right\rangle
$$

Clearly, $\rho_{0}(x)=1$ for all $x \in S^{2}$. By continuity, we can find a positive real number $\delta_{3}<\delta_{2}$ such that $\rho_{t}(x)>0$ for all $x \in S^{2}$ and all $t \in\left(-\delta_{3}, \delta_{3}\right)$. The lapse function $\rho_{t}: S^{2} \rightarrow \mathbb{R}$ satisfies the Jacobi equation

$$
\Delta_{f_{t}^{*} g} \rho_{t}+\left(\operatorname{Ric}\left(\nu_{t}, \nu_{t}\right)+\left|I I_{t}\right|^{2}\right) \rho_{t}=-H^{\prime}(t)
$$

(cf.[10, equation (1.2)]).

Proposition 3.5. We have area $\left(S^{2}, f_{t}^{*} g\right)=4 \pi$ for all $t \in\left(-\delta_{3}, \delta_{3}\right)$.

Proof. Let $\bar{\rho}_{t}$ denote the mean value of the lapse function $\rho_{t}: S^{2} \rightarrow \mathbb{R}$ with respect to the induced metric $f_{t}^{*} g$; that is,

$$
\bar{\rho}_{t}=\frac{1}{\operatorname{area}\left(S^{2}, f_{t}^{*} g\right)} \int_{S^{2}} \rho_{t} d \mu_{f_{t}^{*} g} .
$$

It follows from Lemma 3.3 that

$$
\int_{S^{2}}\left|\nabla \rho_{t}\right|_{f_{t}^{*} g}^{2} d \mu_{f_{t}^{*} g}-\int_{S^{2}}\left(\operatorname{Ric}\left(\nu_{t}, \nu_{t}\right)+\left|I I_{t}\right|^{2}\right)\left(\rho_{t}-\bar{\rho}_{t}\right)^{2} d \mu_{f_{t}^{*} g} \geq 0
$$


for all $t \in\left(-\delta_{2}, \delta_{2}\right)$. Moreover, Lemma 3.4 implies that

$$
\bar{\rho}_{t}^{2} \int_{S^{2}}\left(\operatorname{Ric}\left(\nu_{t}, \nu_{t}\right)+\left|I I_{t}\right|^{2}\right) d \mu \geq 0
$$

for all $t \in\left(-\delta_{1}, \delta_{1}\right)$. Adding both inequalities yields

$$
\int_{S^{2}}\left|\nabla \rho_{t}\right|_{f_{t}^{*} g}^{2} d \mu_{f_{t}^{*} g}+\int_{S^{2}}\left(\operatorname{Ric}\left(\nu_{t}, \nu_{t}\right)+\left|I I_{t}\right|^{2}\right) \rho_{t}\left(2 \bar{\rho}_{t}-\rho_{t}\right) d \mu_{f_{t}^{*} g} \geq 0
$$

for all $t \in\left(-\delta_{2}, \delta_{2}\right)$.

In the next step, we multiply Equation (3.4) by $2 \bar{\rho}_{t}-\rho_{t}$ and integrate. This gives

$$
\begin{gathered}
\int_{S^{2}}\left|\nabla \rho_{t}\right|_{f_{t}^{*} g}^{2} d \mu_{f_{t}^{*} g}+\int_{S^{2}}\left(\operatorname{Ric}\left(\nu_{t}, \nu_{t}\right)+\left|I I_{t}\right|^{2}\right) \rho_{t}\left(2 \bar{\rho}_{t}-\rho_{t}\right) d \mu_{f_{t}^{*} g} \\
=-H^{\prime}(t) \int_{S^{2}}\left(2 \bar{\rho}_{t}-\rho_{t}\right) d \mu_{f_{t}^{*} g}=-H^{\prime}(t) \int_{S^{2}} \rho_{t} d \mu_{f_{t}^{*} g}
\end{gathered}
$$

Putting these facts together, we obtain

$$
H^{\prime}(t) \int_{S^{2}} \rho_{t} d \mu_{f_{t}^{*} g} \leq 0
$$

for each $t \in\left(-\delta_{2}, \delta_{2}\right)$. Therefore, we have $H^{\prime}(t) \leq 0$ for all $t \in\left(-\delta_{3}, \delta_{3}\right)$. Since $H(0)=0$, it follows that $H(t) \geq 0$ for all $t \in\left(-\delta_{3}, 0\right]$ and $H(t) \leq 0$ for all $t \in\left[0, \delta_{3}\right)$. Using the identity

$$
\frac{d}{d t} \operatorname{area}\left(S^{2}, f_{t}^{*} g\right)=\int_{S^{2}}\left\langle H(t) \nu_{t}, \frac{\partial}{\partial t} f_{t}\right\rangle d \mu_{f_{t}^{*} g}=H(t) \int_{S^{2}} \rho_{t} d \mu_{f_{t}^{*} g}
$$

we obtain

$$
\operatorname{area}\left(S^{2}, f_{t}^{*} g\right) \leq \operatorname{area}\left(S^{2}, f^{*} g\right)=4 \pi
$$

for all $t \in\left(-\delta_{3}, \delta_{3}\right)$. Since $f$ minimizes area in its homotopy class, we conclude that area $\left(S^{2}, f_{t}^{*} g\right)=4 \pi$ for all $t \in\left(-\delta_{3}, \delta_{3}\right)$.

Proposition 3.6. For each $t \in\left(-\delta_{3}, \delta_{3}\right)$, the surface $\Sigma_{t}$ is totally geodesic, and we have $R=2$ and $\operatorname{Ric}\left(\nu_{t}, \nu_{t}\right)=0$ at each point on $\Sigma_{t}$. Moreover, the lapse function $\rho_{t}: S^{2} \rightarrow \mathbb{R}$ is constant.

Proof. By Proposition 3.5, we have area $\left(S^{2}, f_{t}^{*} g\right)=4 \pi$. Hence, it follows from Proposition 3.1 that $\Sigma_{t}$ is totally geodesic, and $R=2$ and $\operatorname{Ric}\left(\nu_{t}, \nu_{t}\right)=$ 0 at each point on $\Sigma_{t}$. Substituting this into (3.4), we obtain $\Delta_{f_{t}^{*} g} \rho_{t}=0$. Therefore, the function $\rho_{t}: S^{2} \rightarrow \mathbb{R}$ is constant, as claimed. 
Corollary 3.7. The normal vector field $\nu_{t}$ is a parallel vector field near $\Sigma$. In particular, each point on $\Sigma$ has a neighborhood which is isometric to a Riemannian product.

Proof. By Proposition 3.6, the lapse function $\rho_{t}: S^{2} \rightarrow \mathbb{R}$ is constant. This implies

$$
\begin{aligned}
& \left\langle D_{\frac{\partial f_{t}}{\partial x_{i}}} \nu_{t}, \frac{\partial f_{t}}{\partial t}\right\rangle-\left\langle D_{\frac{\partial f_{t}}{\partial t}} \nu_{t}, \frac{\partial f_{t}}{\partial x_{i}}\right\rangle \\
& =\frac{\partial}{\partial x_{i}}\left\langle\nu_{t}, \frac{\partial f_{t}}{\partial t}\right\rangle-\frac{\partial}{\partial t}\left\langle\nu_{t}, \frac{\partial f_{t}}{\partial x_{i}}\right\rangle=\frac{\partial}{\partial x_{i}} \rho_{t}(x)=0
\end{aligned}
$$

for each point $x \in S^{2}$. Moreover, we have

$$
\begin{aligned}
& \left\langle D_{\frac{\partial f_{t}}{\partial x_{i}}} \nu_{t}, \frac{\partial f_{t}}{\partial x_{j}}\right\rangle-\left\langle D_{\frac{\partial f_{t}}{\partial x_{j}}} \nu_{t}, \frac{\partial f_{t}}{\partial x_{i}}\right\rangle \\
& =\frac{\partial}{\partial x_{i}}\left\langle\nu_{t}, \frac{\partial f_{t}}{\partial x_{j}}\right\rangle-\frac{\partial}{\partial x_{j}}\left\langle\nu_{t}, \frac{\partial f_{t}}{\partial x_{i}}\right\rangle=0
\end{aligned}
$$

for all $x \in S^{2}$. Putting these facts together, we obtain

$$
\left\langle D_{\frac{\partial f_{t}}{\partial x_{i}}} \nu_{t}, V\right\rangle-\left\langle D_{V} \nu_{t}, \frac{\partial f_{t}}{\partial x_{i}}\right\rangle=0
$$

for each point $x \in S^{2}$ and all vectors $V \in T_{f(x)} M$. In particular, we have

$$
\left\langle D_{\frac{\partial f_{t}}{\partial x_{i}}} \nu_{t}, \nu_{t}\right\rangle-\left\langle D_{\nu_{t}} \nu_{t}, \frac{\partial f_{t}}{\partial x_{i}}\right\rangle=0
$$

for each point $x \in S^{2}$. Since the vector field $\nu_{t}$ has unit length, we conclude that $D_{\nu_{t}} \nu_{t}=0$. On the other hand, it follows from Proposition 3.6 that the surfaces $\Sigma_{t}$ are totally geodesic. This implies $D_{\frac{\partial f_{t}}{\partial x_{i}}} \nu_{t}=0$ for each point $x \in S^{2}$. Thus, we conclude that the normal vector field $\nu_{t}$ is parallel. This completes the proof of Corollary 3.7.

We now consider the product $S^{2} \times \mathbb{R}$, where $S^{2}$ is equipped with the induced metric $f^{*} g$. We define a map $\Phi: S^{2} \times \mathbb{R} \rightarrow M$ by $\Phi(x, t)=\exp _{f(x)}$ $(t \nu(x))$. It follows from Corollary 3.7 that the restriction $\left.\Phi\right|_{S^{2} \times(-\delta, \delta)}$ is a local isometry if $\delta>0$ is sufficiently small.

Proposition 3.8. The map $\Phi: S^{2} \times \mathbb{R} \rightarrow M$ is a local isometry.

Proof. We first show that $\left.\Phi\right|_{S^{2} \times[0, \infty)}$ is a local isometry. Suppose this is false. Let $\tau$ be the largest positive real number with the property that $\left.\Phi\right|_{S^{2} \times[0, \tau]}$ 
is a local isometry. We now define a map $\tilde{f}: S^{2} \rightarrow M$ by $\tilde{f}(x)=\Phi(x, \tau)$. Clearly, $\tilde{f}$ is homotopic to $f$; consequently, $\tilde{f}$ represents a non-trivial element of $\pi_{2}(M)$. Moreover, we have area $\left(S^{2}, \tilde{f}^{*} g\right)=\operatorname{area}\left(S^{2}, f^{*} g\right)=4 \pi$. Therefore, $\tilde{f}$ has minimal area among all maps in $\mathscr{F}$. By Corollary 3.7, each point on the surface $\tilde{\Sigma}=\tilde{f}\left(S^{2}\right)$ has a neighborhood which is isometric to a Riemannian product. Hence, if $\delta>0$ is sufficiently small, then the map $\left.\Phi\right|_{S^{2} \times[0, \tau+\delta)}$ is a local isometry. This contradicts the maximality of $\tau$.

Therefore, the restriction $\left.\Phi\right|_{S^{2} \times[0, \infty)}$ is a local isometry. An analogous argument shows that $\left.\Phi\right|_{S^{2} \times(-\infty, 0]}$ is a local isometry. This completes the proof of Proposition 3.8.

Since $\Phi: S^{2} \times \mathbb{R} \rightarrow M$ is a local isometry, it follows that $\Phi$ is a covering map (cf.[6, Section 1.11]). Consequently, the universal cover of $(M, g)$ is isometric to $S^{2} \times \mathbb{R}$, equipped with the standard metric. This completes the proof of Theorem 1.1.

\section{Acknowledgments}

The authors would like to thank Fernando Marques for discussions, and Willie Wong for comments on an earlier version of this paper.

\section{References}

[1] H. Bray, The Penrose inequality in general relativity and volume comparison theorems involving scalar curvature, $\mathrm{PhD}$ thesis, Stanford University (1997).

[2] H. Bray, Proof of the Riemannian Penrose inequality using the positive mass theorem, J. Diff. Geom. 59 (2001), 177-267.

[3] H. Bray, S. Brendle, M. Eichmair, and A. Neves, Area-minimizing projective planes in three-manifolds, Comm. Pure Appl. Math. 63 (2010), $1237-1247$.

[4] S. Brendle, Rigidity phenomena involving scalar curvature, Surveys in Differential Geometry (to appear)

[5] M. Cai and G. Galloway, Rigidity of area minimizing tori in 3-manifolds of nonnegative scalar curvature, Comm. Anal. Geom. 8 (2000), 565-573.

[6] J. Cheeger and D. Ebin, Comparison theorems in Riemannian geometry, American Mathematical Society, Providence, RI (2008).

[7] G. Galloway, Rigidity of marginally trapped surfaces and the topology of black holes, Comm. Anal. Geom. 16 (2008), 217-229. 
[8] F. Hang and X. Wang, Rigidity theorems for compact manifolds with boundary and positive Ricci curvature, J. Geom. Anal. 19 (2009), 628-642.

[9] J. Hass and P. Scott, The existence of least area surfaces in 3-manifolds, Trans. Amer. Math. Soc. 310 (1988), 87-114.

[10] G. Huisken and T. Ilmanen, The inverse mean curvature flow and the Riemannian Penrose inequality, J. Differ. Geom. 59 (2001), 353-437.

[11] G. Huisken and S.T. Yau, Definition of center of mass for isolated physical systems and unique foliations by stable spheres with constant mean curvature, Invent. Math. 124 (1996), 281-311.

[12] W. Meeks and S.T. Yau, Topology of three-dimensional manifolds and the embedding problems in minimal surface theory, Ann. Math. 112 (1980), 441-484.

[13] R. Schoen and S.T. Yau, Existence of incompressible minimal surfaces and the topology of three-dimensional manifolds with nonnegative scalar curvature, Ann. Math. 110 (1979), 127-142.

[14] V. Toponogov, Evaluation of the length of a closed geodesic on a convex surface, Dokl. Akad. Nauk. SSSR 124 (1959), 282-284

Department of Mathematics

DUKE UNIVERSITY

DURHAM, NC 27708

USA

E-mail address: bray@math.duke.edu

Department of Mathematics

STANFORD UNIVERSITY

450 Serra Mall, Bldg 380

STANFORD, CA 94305

USA

E-mail address: brendle@math.stanford.edu

Imperial College

HuXLEY BuILDING

180 QueEn's Gate

LONDON SW7 2RH

UK

E-mail address: a.neves@imperial.ac.uk

Received February 24, 2010 